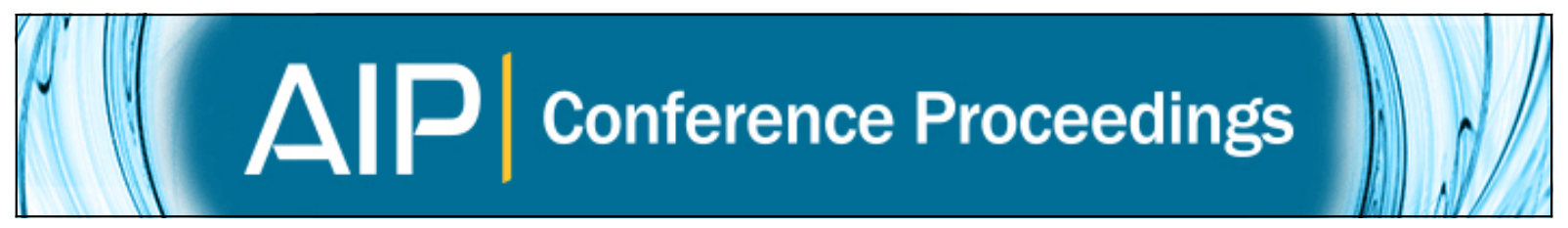

Novel technique for spatially resolved imaging of molecular bond orientations using $x-$ ray birefringence

John P. Sutter, Igor P. Dolbnya, Stephen P. Collins, Kenneth D. M. Harris, Gregory R. Edwards-Gau, Benson M. Kariuki, and Benjamin A. Palmer

Citation: AIP Conference Proceedings 1741, 050009 (2016); doi: 10.1063/1.4952929

View online: http://dx.doi.org/10.1063/1.4952929

View Table of Contents: http://scitation.aip.org/content/aip/proceeding/aipcp/1741?ver=pdfcov

Published by the AIP Publishing

Articles you may be interested in

Resolving hot spot microstructure using x-ray penumbral imaging (invited)

Rev. Sci. Instrum. 87, 11E201 (2016); 10.1063/1.4959161

A novel technique for single-shot energy-resolved $2 \mathrm{D} x$-ray imaging of plasmas relevant for the inertial confinement fusion

Rev. Sci. Instrum. 83, 103504 (2012); 10.1063/1.4759135

Temporal resolved $x$-ray penumbral imaging technique using heuristic image reconstruction procedure and wide dynamic range $\mathrm{x}$-ray streak camera

Rev. Sci. Instrum. 75, 4010 (2004); 10.1063/1.1787900

Novel time and two-dimensional space-resolved $x$-ray imaging technique

Rev. Sci. Instrum. 60, 3558 (1989); 10.1063/1.1140511

The orientation and bonding of $\mathrm{CO}$ on $\mathrm{Mo}(100)$ using angle-resolved photoelectron spectroscopy and near-edge x-ray absorption fine structure

J. Chem. Phys. 87, 7265 (1987); 10.1063/1.453372 


\title{
Novel technique for spatially resolved imaging of molecular bond orientations using $\mathrm{X}$-ray birefringence
}

\author{
John P. Sutter ${ }^{1, \text { a) }}$, Igor P. Dolbnya ${ }^{1}$, Stephen P. Collins ${ }^{1}$, Kenneth D. M. Harris ${ }^{2, b)}$, \\ Gregory R. Edwards-Gau ${ }^{2}$, Benson M. Kariuki ${ }^{2}$, and Benjamin A. Palmer ${ }^{3}$ \\ ${ }^{1}$ Diamond Light Source Ltd, Harwell Science and Innovation Campus, Chilton, Didcot, Oxfordshire OX11 0DE, \\ United Kingdom \\ ${ }^{2}$ School of Chemistry, Cardiff University, Park Place, Cardiff CF10 3AT, United Kingdom \\ ${ }^{3}$ Department of Structural Biology, Weizmann Institute of Science, 234 Herzl St., Rehovot 7610001, Israel \\ ${ }^{\text {a)} C o r r e s p o n d i n g ~ a u t h o r: ~ j o h n . s u t t e r @ d i a m o n d . a c . u k ~}$ \\ ${ }^{b)}$ Corresponding author: HarrisKDM@cardiff.ac.uk
}

\begin{abstract}
Birefringence has been observed in anisotropic materials transmitting linearly polarized X-ray beams tuned close to an absorption edge of a specific element in the material. Synchrotron bending magnets provide X-ray beams of sufficiently high brightness and cross section for spatially resolved measurements of birefringence. The recently developed X-ray Birefringence Imaging (XBI) technique has been successfully applied for the first time using the versatile test beamline B16 at Diamond Light Source. Orientational distributions of the $\mathrm{C}-\mathrm{Br}$ bonds of brominated "guest" molecules within crystalline "host" tunnel structures (in thiourea or urea inclusion compounds) have been studied using linearly polarized incident X-rays near the $\mathrm{Br} \mathrm{K}$-edge. Imaging of domain structures, changes in $\mathrm{C}-\mathrm{Br}$ bond orientations associated with order-disorder phase transitions, and the effects of dynamic averaging of $\mathrm{C}-\mathrm{Br}$ bond orientations have been demonstrated. The XBI setup uses a vertically deflecting high-resolution double-crystal monochromator upstream from the sample and a horizontally deflecting single-crystal polarization analyzer downstream, with a Bragg angle as close as possible to $45^{\circ}$. In this way, the ellipticity and rotation angle of the polarization of the beam transmitted through the sample is measured as in polarizing optical microscopy. The theoretical instrumental background calculated from the elliptical polarization of the bending-magnet X-rays, the imperfect polarization discrimination of the analyzer, and the correlation between vertical position and photon energy introduced by the monochromator agrees well with experimental observations. The background is calculated analytically because the region of X-ray phase space selected by this setup is sampled inefficiently by standard methods.
\end{abstract}

\section{INTRODUCTION}

Birefringence (also called double refraction) is observed when linearly polarized visible light is transmitted through a non-cubic crystal. Recently, crystals of certain compounds have been found to show birefringence of Xrays tuned to an absorption edge of one of the types of atom present in the material [1,2]. While optical birefringence arises from the global anisotropy of the crystal, X-ray birefringence arises from the locally anisotropic environment of the selected type of atom, and may therefore be used to study the orientational properties of molecules and bonds. X-rays tuned to the $\mathrm{Br} \mathrm{K}$ edge (13.474 keV [3]) have been used to examine the orientational properties of the $\mathrm{C}-\mathrm{Br}$ bonds of brominated organic molecules included as "guests" in thiourea and urea "host" crystals. The earliest studies were performed with narrowly focused X-ray beams, but with the introduction of X-ray Birefringence Imaging (XBI) using bending-magnet beams of large cross section, broad bandwidth and strong linear polarization, spatially resolved mapping of the orientation of the X-ray optic axis in domains within the sample was demonstrated $[4,5]$. A setup for XBI with a resolution of $10 \mu \mathrm{m}$ has been developed and tested at the versatile bending-magnet test beamline B16 at Diamond Light Source. The XBI data yielded many new structural insights 
about the materials under investigation, but the images are found to be superimposed on an instrumental background. The sources of this background are discussed at length by Sutter et al [6] and are summarized here.

Crystals of urea and thiourea inclusion compounds contain tunnels along their $c$-axis, within which brominated organic guest molecules may be accommodated (see FIGURE 1). In general, thiourea inclusion compounds are rhombohedral at ambient temperature and monoclinic below a low-temperature phase transition (although 1bromoadamantane/thiourea does not exhibit a phase transition). Urea inclusion compounds are usually hexagonal at ambient temperature and orthorhombic below a low-temperature phase transition. The phase transition temperature depends on the type of guest molecule. Absorption of X-rays tuned to the $\mathrm{Br} \mathrm{K}$ edge is highly sensitive to the orientation of the $\mathrm{C}-\mathrm{Br}$ bonds because such X-rays excite dipole transitions to $p$-like antibonding orbitals. As a result, these inclusion compounds show intense linear X-ray dichroism and X-ray birefringence (these phenomena are connected by a Kramers-Kronig relation). The birefringence of most of these materials is uniaxial.

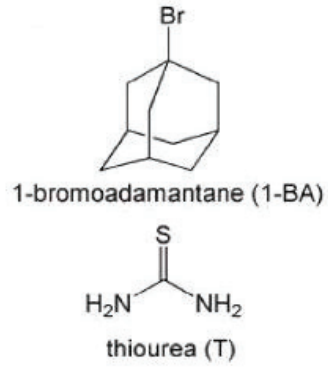

(a)

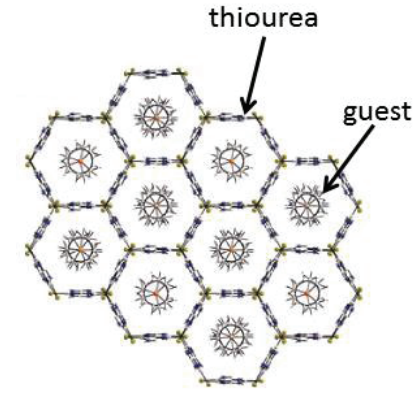

(b)

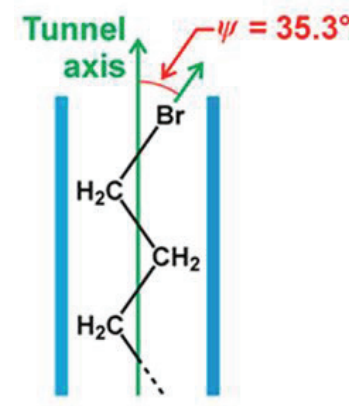

(c)

FIGURE 1. (a) Molecular structures of one type of brominated organic guest molecule (1-bromoadamantane; 1-BA) and thiourea. In urea, the sulfur atom in thiourea is replaced by oxygen. (b) Crystal structure of the 1-BA/thiourea inclusion compound viewed along the thiourea tunnel axis (the $\mathrm{C}-\mathrm{Br}$ bonds of the 1-BA guest molecules are oriented along the tunnel). (c)

For $\alpha, \omega$-dibromoalkane guest molecules in the urea host structure, the $\mathrm{C}-\mathrm{Br}$ bonds are at $35.3^{\circ}$ from tunnel axis.

The experimental setup for XBI is displayed in FIGURE 2. The X-rays from the bending magnet are polarized to a high degree in the horizontal plane. The vertically deflecting double-crystal monochromator is symmetric $\mathrm{Si}$ (1 11 1). The beam covers the entire sample, which is mounted on the Huber diffractometer of B16 for accurate angular alignment. In the experiments discussed here, the crystal was oriented so that the $c$-axis lies in the plane perpendicular to the beam direction. The horizontally deflecting polarization analyzer is either a $\mathrm{Si}(555)$ or a $\mathrm{Ge}$ ( 55 5) single crystal. The Bragg reflection is symmetric. At the $\mathrm{Br} \mathrm{K}$ edge, the Bragg angle $\theta_{B}$ of the analyzer is $47.193^{\circ}$ or $44.768^{\circ}$, respectively. By deflecting the beam horizontally by an angle $2 \theta_{B}$ close to $90^{\circ}$, the analyzer rejects the main horizontal component of polarization of the incident beam. Therefore, in principle, any X-rays transmitted to the area detector (the camera) must have had their polarization rotated by the sample, thus indicating that the sample is birefringent. This method is analogous to polarizing optical microscopy using visible light.

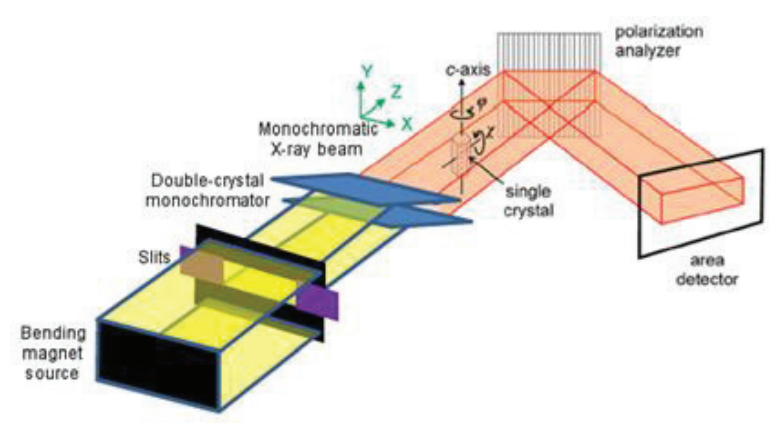

(a)

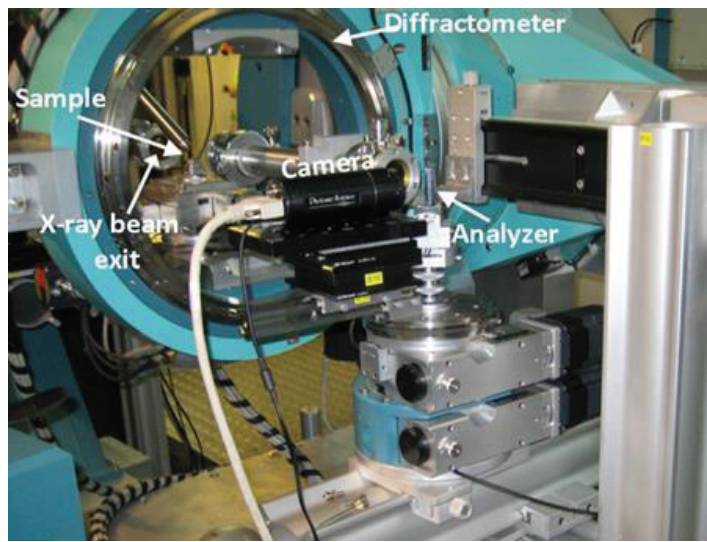

(b)

FIGURE 2. (a) Schematic of the X-ray Birefringence Imaging setup on the versatile test beamline B16 at Diamond. (b) Photograph of the X-ray Birefringence Imaging setup in (a) excluding the double-crystal monochromator. 
Examples of XBI data recorded at B16 are shown in FIGURE 3. Images of the X-rays transmitted through the setup in FIGURE 2 are recorded by the camera as the sample is rotated through $\chi$ about the direction of the incident beam. At $\chi=0^{\circ}$ the $c$-axis lies in the horizontal plane. The X-ray birefringence is shown by the bright region of the image that becomes strongest at $\chi=45^{\circ}$. However, the bright region appears on a background of complex shape consisting of a tilted stripe with a dark band cutting horizontally through the middle. This is clearly not part of the data from the sample and must be understood in greater detail if the experimental data are to be interpreted correctly.

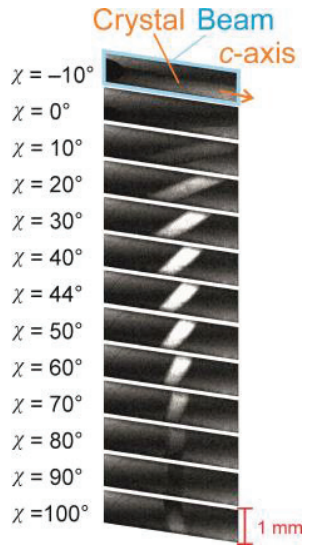

(a)

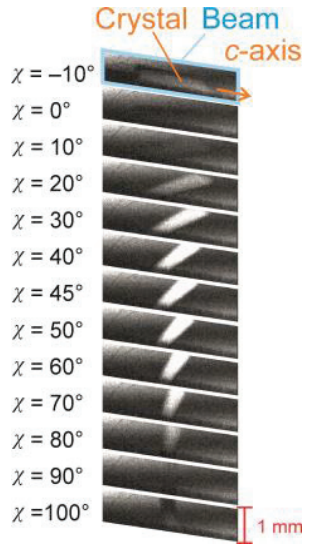

(b)

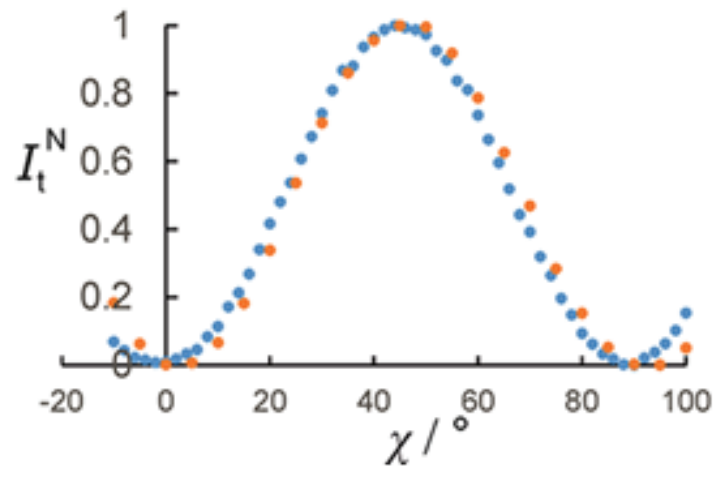

(c)

FIGURE 3. XB images [recorded using a Ge (5 55 ) analyzer] showing the transmitted beam as a function of angle $\chi$ between the horizontal plane and the $c$-axis for: (a) a single crystal of 1,8-dibromooctane/urea at $280 \mathrm{~K}$, and (b) a single crystal of 1,10dibromodecane/urea at $170 \mathrm{~K}$. (c) Variation of normalized intensity with $\chi$ [left-shifted blue: data from (a); right-shifted orange: data from (b)].

The XBI setup used at Diamond falls short of the ideal in three ways. First, the X-rays produced by bending magnet sources are linearly horizontally polarized only in the plane of the electron orbit. Above and below this plane, the X-rays are elliptically polarized to a degree that depends on the electron energy $(3 \mathrm{GeV})$, the bending radius $(6.981 \mathrm{~m})$ and the vertical angle [7]. Detailed ray traces applying the software package SHADOW [8] to an accurate model of the electron beam in the Diamond storage ring show that the horizontally polarized intensity has a single peak centred at zero vertical angle with a vertical FWHM of $200 \mu \mathrm{rad}$, while the vertically polarized intensity has two peaks at vertical angles $\pm 100 \mu \mathrm{rad}$ and falls to zero at zero vertical angle as expected. Second, the photon energy matching the selected absorption edge generally cannot be diffracted through a deflection angle of exactly $90^{\circ}$ by any Bragg reflection of the crystal analyzer. Therefore, even the analyzer Bragg reflection that comes closest will not completely suppress the horizontal polarization. Detailed calculations of the intensity diffracted by the analyzer have been made using dynamical diffraction theory [9]. If $\theta_{1}$ and $\theta_{2}$ are, respectively, the Bragg angles of the monochromator and the analyzer, and if $k_{0}$ is the wave vector at which the Bragg condition is fulfilled at $\theta_{1}$ and $\theta_{2}$, then parameters $A_{1}$ and $A_{2}$ describing the deviation from the Bragg condition of a ray with wave number $k$ incident on the monochromator at grazing angle $\theta_{i 1}$ or on the analyzer at grazing angle $\theta_{i 2}$ can be defined [10] as

$$
A_{1,2}=4\left(\frac{k_{0}}{k}\right) \sin \theta_{1,2}\left[\left(\frac{k_{0}}{k}\right) \sin \theta_{1,2}-\sin \theta_{i 1,2}\right] .
$$

The rocking curves of the Bragg reflections differ depending on whether the incident X-rays are polarized perpendicular to the plane of the incident and diffracted beams (s-polarization) or parallel to that plane $(p$ polarization). Ideally the $p$ rocking curve of the analyzer would be zero, but in reality it must be accurately calculated for a full understanding of the background. Therefore $R_{\mathbf{H} 1 \mathrm{~s}}\left(A_{1}\right)$ and $R_{\mathbf{H} 1 \mathrm{p}}\left(A_{1}\right)$, the $s$ and $p$ rocking curves of the monochromator, and $R_{\mathbf{H} 2 \mathrm{~s}}\left(A_{2}\right)$ and $R_{\mathbf{H} 2 \mathrm{p}}\left(A_{2}\right)$, the $s$ and $p$ rocking curves of the analyzer, have been calculated. Third, because the Bragg angle depends on the photon energy, the diffracted beam from the monochromator has an angular dispersion of photon energy in the vertical direction, and in turn the analyzer imposes an angular dependence in the horizontal direction on the photon energies that it diffracts. If a given ray from the bending magnet is defined by its horizontal angle $\alpha$, vertical angle $\beta$ and wave number $k_{0}+\delta k$, then on a 3-dimensional plot with $\alpha, \beta$ and $\delta k$ axes the subset of rays that reach the camera is represented by a thin volume enclosing a line that is 
not parallel to any of these axes. We note that ray traces that sample rays within a rectangular region with edges parallel to these axes are therefore inefficient. The orientation of the line is given by $\beta=\alpha\left(\tan \theta_{1} / \tan \theta_{2}\right)$ and $\delta k / k=-$ $\beta \cot \theta_{1}=-\alpha \cot \theta_{2}$. To determine the instrumental background on the camera, the bending magnet X-ray intensity in the horizontal and vertical directions is multiplied by the appropriate rocking curves. The product is integrated over all rays with the same $\alpha$ and $\beta$ that are transmitted by both the monochromator and the analyzer. Theoretical calculations and experimental measurements, shown in FIGURE 4, are clearly in good agreement.

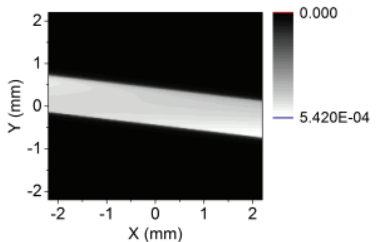

(a)

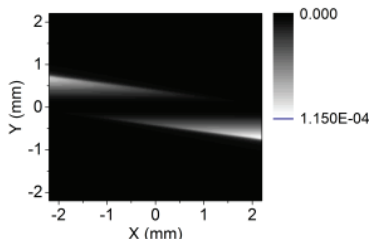

(b)

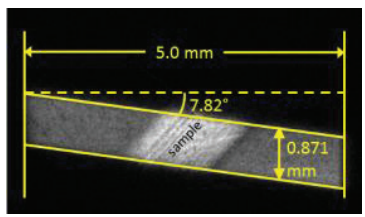

(c)

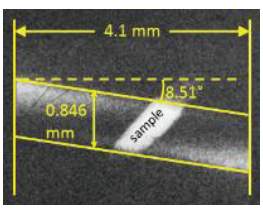

(d)

FIGURE 4. Calculated total intensity on the camera using: (a) the Si (5 5 5) analyzer, and (b) the Ge (5 5 5) analyzer. Measured images on the camera using: (c) the $\operatorname{Si}\left(\begin{array}{l}5 \\ 5\end{array}\right.$ ) analyzer, and (d) the Ge (5 5 5) analyzer. (c) includes a birefringent single crystal sample of 1-bromoadamantane/thiourea and (d) includes a birefringent single crystal sample of 1,8-dibromooctane/urea.

X-ray Birefringence Imaging has thus been demonstrated at the versatile test beamline B16 at Diamond Light Source, and has been shown to allow mapping of the local orientational properties of specified molecules and/or bonds in anisotropic materials. The instrumental background is considerable and is difficult to model with standard ray sampling, but nevertheless it can be readily explained, as discussed above.

\section{ACKNOWLEDGMENTS}

The authors thank Ian Martin and Riccardo Bartolini of the Accelerator Physics department at Diamond for their accurate description of the electron beam in the Diamond storage ring, and Manuel Sánchez del Río of the ESRF for his patient assistance with SHADOW. The EPSRC provided a studentship to one of the authors (Edwards-Gau) and Cardiff University provided financial support for this project. Benjamin Palmer is currently being supported by a Human Frontiers Fellowship from the Human Frontiers Science Program. Kawal Sawhney provided assistance with beamline B16 at Diamond and Andrew Malandain provided technical support. This work was carried out with the support of Diamond Light Source under proposal MT8323-1.

\section{REFERENCES}

1. B. A. Palmer, A. Morte-Ródenas, B. M. Kariuki, K. D. M. Harris and S. P. Collins, J. Phys. Chem. Lett. 2, 2346-2351 (2011).

2. B. A. Palmer, G. R. Edwards-Gau, A. Morte-Ródenas, B. M. Kariuki, G. K. Lim, K. D. M. Harris, I. P. Dolbnya and S. P. Collins, J. Phys. Chem. Lett. 3, 3216-3222 (2012).

3. J. A. Bearden and A. F. Burr, Rev. Mod. Phys. 39, 125-142 (1967).

4. B. A. Palmer, G. R. Edwards-Gau, B. M. Kariuki, K. D. M. Harris, I. P. Dolbnya and S. P. Collins, Science 344, 1013-1016 (2014).

5. B. A. Palmer, G. R. Edwards-Gau, B. M. Kariuki, K. D. M. Harris, I. P. Dolbnya, S. P. Collins and J. P. Sutter, J. Phys. Chem. Lett. 6, 561-567 (2015).

6. J. P. Sutter, I. P. Dolbnya, S. P. Collins, K. D. M. Harris, G. R. Edwards-Gau and B. A. Palmer, J. Appl. Phys. 117, 164902 (2015).

7. J. D. Jackson, Classical Electrodynamics, $2^{\text {nd }}$ edition (John Wiley \& Sons, New York, 1975), pp. $672-679$.

8. M. Sánchez del Río, N. Canestrari, F. Jiang and F. Cerrina, J. Synchrotron Rad. 18, 708-716 (2011).

9. W. H. Zachariasen, Theory of X-Ray Diffraction in Crystals (John Wiley \& Sons, New York, 1945), pp. 82147.

10. Yu. V. Shvyd'ko, E. Gerdau, J. Jäschke, O. Leupold, M. Lucht and H. D. Rüter, Phys. Rev. B 57, 4968-4971 (1998). 